aide aux malades de la Saxe qu'ont été employées les infirmières albertines. Nous ne pouvons pas suivre l'activité de la Société dans ce domaine, qui n'est pas celui de la Croix-Rouge; ajoutons seulement qu'à la fin de 1885, le nombre des Albertines en activité s'élevait à 113. Sur ce nombre, 22 seulement se trouvaient dans le Carolahaus, les autres étaient réparties dans d'autres établissements hospitaliers de Dresde ou dans d'autres localités. Depuis 1878, époque de la fondation du Carolahaus, jusqu'à la fin de 1885, il avait été soigné 3917 malades, sans compter ceux qui avaient été traités daus les policliniques.

Dès sa fondation, la Société a compris la nécessité de créer des Sociétés filiales, et elle s'est efforcée d'en provoquer l'organisation dans toute la Saxe. C'est ainsi qu'à la fin de 1885 on en comptait 38, qui, dans toutes les parties du pays, s'occupent à soigner les malades.

Enfin, la Société possède des établissements hospitaliers à Leipzig, à Grunau et à Dresde. C'est dans cette dernière ville qu'a été ouvert en 1878 l'hôpital Carola, le plus considérable et le plus important de tous; il a déjà rendu de nombreux services et on espère qu'il en rendra encore davantage, lorsque les agrandissements qu'on se propose de lui faire subir auront pu être exécutés.

\title{
SUISSE
}

\section{LA SOCIÉTÉ SUISSE DE 1884 A 1886}

\section{Extrail du rapport de la Direction.}

Si nous jetons un coup d'œil en arrière sur la courte carrière de la Société centrale suisse de la Croix-Rouge, nous devons malheureusement constater qu'elle n'a pas, dans son développement, suivi une marche aussi ascensionnelle, et n'a pas oblenu tous les résultats que faisaient espérer l'entrain juvénile dont elle était. animée lors de sa fondation et les principes élevés proclamés par ses statuts. Il est vrai que ce ne sont pas, du moins dans beau- 
coup de localités, les démonstrations et les avis encourageants, ni les adhésions empressées, qui lui ont fait défaut, mais divers obstacles se sont opposés à ce qu'elle prît une extension vigoureuse. A notre époque, si fertile en associations, tellemeut qu'on commence à en être las, toute nouvelle association mise sur le tapis, quelles que soient l'opportunité de son existence et la noblesse de ses efforts, a d'abord à lutter péniblement contre des sœurs et des demi-sœurs plus anciennes, pour assurer son droil à l'existence et arriver à se faire reconnaître. D'autre part, l'organisation primitive et la répartition des tàches de la Société furent reconnues défectueuses; les buts divers qu'elle se proposait d'atteindre n'étaient peut être pas assez bien définis; les uns étaient conçus d'une manière trop générale, les autres manquaient de précision, en sorte que la Direction, dans ses différentes séances et délibérations, eut à se préoccuper avant tout, outre la question de propagande, d'établir une délimitation plus fixe, plus précise et plus pratique de ses devoirs.

Les nombreuses ef mures délibérations qui eurent lieu, notamment depuis 1885 , ont eu pour résultat la rédaction de nouveaux statuts qui, sur notre proposition, ont été adoptés par la dernière assemblée générale d'Aarau, el qui ont élé envoyés avec le présent rapport à tous les membres de la Société ${ }^{1}$. Nous espérons que ce nouvel état de choses imprimera une impulsion nouvelle à l'activité de la Direction, et qu'elle s'élancera avec entrain dans la voie qui lui est tracée. Nous espérons aussi qu'en tenant compte de ce que le but de la Société est plus clairement défini et de ce que, grâce à la réorganisation effectuée, la marche des affaires de la Société sera plus régulière, non seulement nos membres actuels resteront fidèles au drapeau de la Croix-Rouge, mais aussi une foule de nouveaux membres viendront promptement se grouper autour de lui. On peut d'autant mieux s'y attendre, que la direction des affaires de la Société a pu être confiée à un homme qui inspire pleine confiance, qui possède le jugement, l'habileté et l'énergie nécessaires, qui peut disposer d'un temps suffisant et qui jonit d'une position sociale éminente, en sorte qu'il peut à juste titre assumer la responsabilité qu'impose cette charge. Nous sommes d'autant plus heureux d'avoir cet homme à notre tête,

Voy, ci-dessous, p. 34. 
que, sous la direction de M. Kempin, le fondateur et le premier président de la Société, par suite de diverses circonstances, la Société de la Croix-Rouge suisse paraissait subir un mouvement de recul désastreux et presque marcher à une dissolution regrettable.

Sur la présentation unanime du Comité directeur, l'Assemblée générale, tenue à Aarau le 22 novemble 1886, a élu M. le $\mathrm{D}^{\mathrm{r}} \mathrm{A}$. Stähelin, d'Aarau, aux fonctions de président de la Société et du Comité directeur; ce dernier a été complété par l'adjonction de quelques nouveaux membres.

Depuis la première assemblée générale, qui s'est réunie à Lucerne en décembre 1883 et sur laquelle un rapport a été publié en son temps ${ }^{1}$, il a été tenu, outre un certain nombre de séances du Comité directeur, deux assemblées générales : le 8 décembre 1884 à Berne" et le 22 novembre 1886 à Aarau. Parmi les objets mis en délibération, nous relevons les suivants : Règlement des comptes annuels (solde pour 1882: 260 francs; pour 1883: 503 francs; pour 1884: 771 francs; pour 1885: 1668 fr. 25); rapport sur les résultats de la propagande (en 1884, le nombre des membres s'élevait à 45 sociétés et 394 personnes; en 1885, à 830 sociétés et individus). En remplacement de M. le professeur Aeby, qui est parti et a été nommé membre d'honneur, M. le conseiller d'Etat E. de Steiger, de Berne, a été nommé, en 1884, membre de la Direction. La charge de questeur, qui jusqu'alors avait été assez malencontreusement réunie à celle de président, a été confiée à M. le $D^{r}$ Pestalozzi-Pfyffer, à Brunnen. La Direction s'est fait représenter à la Conférence internationale de Genève, en 1884, par son président d'alors et par le délégué du Conseil fédéral, M. le $D^{r}$ Ziegler, médecin en chef de l'armée. Ces deux délégués ont présenté, à l'assemblée annuelle de Berne, un rapport détaillé sur leur mission et sur les observations qu'ils avaient faites à Genève.

La question soit de la création de cours de Samaritains, soit de l'appui qu'il y aurait lieu de leur fournir et de leur résultats, a attiré à plusieurs reprises l'altention de la Direction, qui s'en est occupée d'une manière approfondie; celle-ci n'a pas eu d'objection à accorder un modeste subside à celte institution. La Direc-

1 Voy. Bulletin, T, XV, p. 118.

2 Voy. T. XVI, p. 40. 
tion croit cependant se conformer au but principal de la Société, qui est de former un fonds de secours sanitaires pour le cas de guerre, en s'abstenant, autant que possible, de prendre des engagements financiers, bien que les demandes à cet effet ne manquent pas. Enfin, en vue d'augmenter le nombre des membres de la Société, il a été décidé de pousser activement la propagande dans les cantons, et, toutes les fois que cela sera possible, de fonder des Sociétés cantonales. Ce sera un des premiers et des plus importants devoirs qui s'imposeront prochainement à la nouvelle Direction élue à Aarau. Les autres questions relatives au but de la Société se présenteront peu à peu d'elles mêmes et seront étudiées au fur et à mesure, en tenant compte des institutions des autres pays et des expériences qui y ont été faites. La Société, en se développant avec une vie nouvelle, va se trouver en face de beaucoup de devoirs à remplir et de relations à établir, par exemple : avec les autorités fédérales, avec les chefs de l'armée, avec le Comité international et avec ses actives congénères, les Sociétés sanitaires militaires; autant de questions qui doivent être réservées pour un prochain rapport.

Enfin, nous faisons savoir que l'Assemblée générale de 1886 , en procédant à la révision des statuts, a composé le Comité directeur de la manière suivante:

MM. Dr A. Stähelin, à Aarau, président.

H. Haggenmacher, avocat, à Zürich, vice-président.

Dr E. Pestalozzi-Pfyffer, à Brunnen, questeur.

R. Wernly, pasteur, à Aarau, secrétaire.

$\mathrm{D}^{\mathrm{r}}$ Ziegler, médecin en chef, à Berne, (membre de droit).

$D^{r}$ Göldli, colonel, à Fluelen.

Dr Kummer, médecin de division, à Aarwangen.

E. de Steiger, conseiller d'Etal, à Berne.

Dr Appia, membre du Comité international, à Genève. ${ }^{1}$ von $\mathrm{Ah}$, pasteur, à Kerns.

Un onzième membre doit encore être nommé par le Comité directeur.

1 M. le Dr Appia a cru devoir refuser de faire partie du Comité central suisse, estimant cette qualité incompatible avec celle de membre du Comité international. 
Quelque sombre et incertaine que paraisse la situation politique actuelle, nous pouvons cependant terminer en exprimant l'espoir qu'il sera donné à la Groix-Rouge de faire flotter longtemps encore son drapeau en temps de paix; mais, si une guerre venait à éclater, puisse-t-elle trouver prêtes, dans notre patrie, ces armes qui guérissent les blessures: les armes de la charité.

Décembre 1886.

La Direction.

STATUTS DE LA SOCIÉTÉ GENTRALE SUISSE DE LA CROIX-ROUGE ${ }^{1}$

\section{$\S 1$}

La Société centrale suisse de la Croix-Rouge a pour but d'organiser et, autant que possible, d'utiliser l'assistance volontaire fournie en temps de guerre au service sanitaire de l'armée.

\section{$\S 2$}

La Société s'efforcera d'atteindre ce but en temps de paix par les moyens suivants:

a) Constitution d'un fonds capital.

b) Formation d'un personnel sanitaire.

c) Etudes statistiques sur le personnel et le matériel sanitaires existant déjà, et acquisition de matériel sanitaire et d'articles de pansement.

d) Etudes sur les localités propres à l'établissement de lazarets, et installation de ceux-ci.

e) Encouragements et appui donnés aux Sociétés ayant pour but de soigner les malades, aux sociétés sanitaires et aux sociétés de secours (par exemple les sociétés samaritaines), qui sont aptes et disposées à concourir, en cas de guerre, à l'ouvre de la Croix-Rouge.

\section{$\S 3$}

En cas de guerre, la Société centrale suisse de la Croix-Rouge se met à la disposition du Conseil fédéral. Elle prend toutes les mesures nécessaires, pour le cas où elle serait désignée par celui-ci comme organe officiel central de l'assistance sanitaire volontaire.

(1) Ces nouveaux statuts, adoptés par l'assemblée générale réunie a Aarau le 22 novembre 1886, remplacent ceux publiés dans le Bulletin, tome XIIl, p. 223. 